\title{
PROPIEDAD INTELECTUAL Y DISTORSIÓN DE LA COMPETENCIA: EL CASO DE ESTADOS UNIDOS*
}

\author{
Mario de la Puente(1), Laura de la Puente(2)
}

INTELLECTUAL PROPERTY AND COMPETITION

DISTORTION: THE CASE OF THE UNITED STATES

PROPRIEDADE INTELECTUAL E DISTORÇÃO DA

COMPETÊNCIA: O CASO DOS ESTADOS UNIDOS

Fecha de recepción: 10 de febrero de 2016

Fecha de aprobación: 23 de junio de 2016

\section{Sugerencia de citación:}

De la Puente, M. \& De la Puente, L. (2017). Propiedad intelectual y distorsión de la competencia: el caso de Estados Unidos. Razón Crítica, 2, 133-159, doi: http://dx.doi.org/10.21789/25007807.1158

* El presente artículo de investigación hace parte del resultado de investigación de la tesis doctoral Economía del turismo de salud: caso colombiano dirigida por el profesor Miguel Cuerdo de la Universidad Rey Juan Carlos, Madrid, España. Con apoyo presupuestario de la Universidad del Norte, Barranquilla, Colombia.

(1) Doctor en Análisis Económico Internacional de la Universidad Rey Juan Carlos de Madrid, España. Magister en Relaciones Económicas Internacionales de la Universidad Rey Juan Carlos, Internacionalista de la Universidad del Norte, Barranquilla, Colombia. Profesor del Departamento de Ciencia Política y Relaciones Internacionales. Miembro del grupo de investigación Agenda Internacional acreditado ante Colciencias. http://orcid.org/0000-0002-0783-1267 Correo electrónico: Mdelapuente@uninorte.edu.co

(2) Estudiante de Maestría en Gerencia Empresarial en la Universidad Rafael Belloso Chacín, Zulia, Venezuela. Profesional en Comunicación Social y Periodismo de la Universidad del Norte. Coordinadora de estrategia comercial de intangibles para Norteamérica en Trading Group International Inc. Asistente de investigación de propiedad intelectual del Departamento de Administración de la Universidad Rafael Belloso Chacín, Zulia, Venezuela.

Correo electrónico: Ldelapuente@urbe.ve 


\section{R E S U M E N}

En este artículo se analizan las patentes como instrumentos de distorsión del principio de libre mercado para el caso de Estados Unidos desde una perspectiva filosófico-económica a partir de un análisis hermenéutico que se complemente con instrumentos cuantitativos. Se encuentran impactos negativos en materia de innovación, investigación y desarrollo de actores económicos para el desarrollo, distribución, comercialización e incluso consumo de bienes por incertidumbre jurídica sobre el permiso de utilización de un invento, así como las limitaciones logísticas que impiden una mayor rigurosidad en el análisis y procedimiento de solicitudes de patentes.

PALABRAS CLAVE: competencia, patente, eficiencia. 


\section{A B S T R A C T}

This paper analyzes patents as distortion instruments of the free market principle using the case of the United States from a philosophic-economical perspective with a hermeneutic analysis complemented with quantitative instruments. Negative impacts are found in the fields of innovation, research and development of economic participants for the development, distribution, commercialization and even consumption of goods due to legal uncertainty regarding the permits for use of an invention, as well as the logistic limitations that prevent a wider strictness in the analysis and procedure of patent inquiries.

KEY WORDS: competition, patents, efficiency.

\section{R E S U M O}

Desde uma perspectiva filosófico-econômica, e com base numa análise hermenêutica complementada por ferramentas quantitativas, analisam-se as patentes como instrumentos de distorção do princípio de livre mercado nos Estados Unidos. Encontraram-se impactos negativos sobre o desenvolvimento de agentes econômicos, a pesquisa e a inovação, afetando especificamente a produção, distribuição, comercialização e consumo de bens. Isto é devido à insegurança jurídica na permissão para usar as invenções, bem como a limitações logísticas que impedem um maior rigor na análise e processamento das aplicações de patentes.

PALAVRAS-CHAVE: competência, patente, eficiência. 


\section{N T R O D U C C I Ó N :}

\section{U N A N Ál I S IS H I S T ÓR I C O}

\section{PARA ESTA D O S U N I D O S}

La finalidad de la competencia es asegurar el funcionamiento lo más eficientemente posible del mercado caracterizado por las interacciones de información a partir del intercambio de bienes y servicios. Desde una perspectiva económica, las prácticas anticompetitivas se fundamentan en el acaparamiento del mercado utilizando instrumentos privativos exclusivos y/o la utilización de ventajas que profundizan la competencia imperfecta aun cuando, desde la perspectiva jurídica, los instrumentos privativos y/o las ventajas utilizadas no sean contrarios a la legalidad. En ese sentido, el derecho de propiedad intelectual a partir del otorgamiento de patentes por concepto de utilidad y/o creación de un invento y/o procedimiento en muchos casos cuestionables por su percepción de obviedad (WIPO, 2014), distorsiona el mercado en materia de precios a través de cambios en los costos totales de elaboración de un bien y utilización de un procedimiento limitando el acceso de éste al nicho de mercado objetivo a través de una discriminación de precios y/o limitaciones legales (CDIP, 2011). 
Lo anterior provoca un conflicto filosófico entre el derecho de propiedad intelectual único del sujeto y el derecho a la libre competencia (Hayek, 1948). La legislación de patentes exige luchar contra copias e imitaciones de productos patentados salvaguardando el derecho al mantenimiento y reconocimiento de la propiedad intelectual (Patent Act, 1793), fundamental en una economía de mercado, mientras que la libre competencia se caracteriza por una intervención mínima del Estado en la planeación, producción, distribución y comercialización de bienes por parte de agentes creativos de la economía (Plant, 1974, p. 24).

El sistema de patentes se basa en unos principios fundamentales fijados con el fin de promover la innovación al otorgar derechos por utilidad y creación que revisten con más probabilidad de interés para el público en general, que protegen las invenciones mas no los descubrimientos y fijan condiciones de patentabilidad de modo que no se puedan conceder patentes a invenciones que carezcan de innovación (Penrose, 1974, p. 40). Según Lemley (2001) existen demasiadas patentes en diversos sectores productivos que resultan en distorsiones de mercado a través de litigios por desconocimiento de un tercero de su existencia y violación al derecho de propiedad intelectual. Se destaca la decisión de la Corte Suprema de Justicia de Estados Unidos en el caso Alice Corp. v. CLS Bank International, 573 US, 134 S.Ct. 2014) 2347) sobre la elegibilidad de patentes para ideas abstractas aplicadas a transacciones financieras electrónicas, en la cual solo se puede patentar un software electrónico si cuenta con un componente o proceso innovador que no exista en sistemas electrónicos financieros disponible en el mercado. Esto ha desincentivado nuevos litigios por patentes en el segmento de software (Allison, Lemley y Shwartz, 2014).

Sin embargo, el sistema de patentes se ha tomado como una oportunidad de ingresos derivados del otorgamiento de licencias a terceros que quieran utilizar un invento o procedimiento, y también como una oportunidad de litigios que incentiva múltiples demandas hacia empresas que (en varios casos) con información limitada de la existencia de un producto patentado ya activo en un mercado, se ven sumergidos en litigios interminables que desincentivan la producción nacional y perpetúan la incertidumbre para futuras creaciones. Este es el caso de Estados Unidos donde la creciente 
producción de bienes, procesos y servicios (en muchos casos poco innovadores) con sus respectivas patentes perjudica la creación, producción y lanzamiento al mercado de nuevos utensilios por temor a litigios, culminando en el pago de licencias que aumentan los precios finales de los bienes y servicios comercializables o la exclusión del producto del mercado limitando la posibilidad de elección de consumidores (CDIP, 2011).

Desde la época colonial los inventores de productos considerados de gran utilidad recurrían a los gobiernos de sus colonias con el fin de garantizar el uso exclusivo del producto o proceso. La primera garantía de derecho de uso exclusivo fue dada en la colonia de Massachusetts en 1641 a Samuel Winslow en el proceso de elaboración de sal por diez años siendo extraoficialmente conocida como la primera patente en Norteamérica (Mokyr, 1990).

Así, varias colonias otorgaban derechos de propiedad sobre bienes y/o procesos generalmente a personas con intereses económicos que involucraban a los representantes colonos. A finales del siglo XVIII las colonias adoptaron normas similares para el otorgamiento de derechos de propiedad intelectual que estandarizó procedimientos de solicitud y otorgamiento de patentes siendo Carolina del Sur el primer Estado en aprobarla en 1784 (Plant, 1974, p. 24) caracterizada por el otorgamiento de derecho de explotación de un procedimiento o artículo por 14 años (PWC, 2014).

Sin embargo, al existir diferencias fundamentales en la normativa comparada existía riesgo de violación de patentes en otros Estados aumentando los costos de protección de derechos de propiedad intelectual (Watson, 2013).

En 1787 Estados Unidos mediante la Constitución Nacional adopta leyes inclusivas de derechos de protección de propiedad intelectual vía patentes a través del aseguramiento exclusivo de derecho de explotación de un invento, procedimiento o descubrimiento por un periodo de tiempo determinado (US Copyright Office, 2008).

En 1790 se aprueba el primer estatuto nacional de patentes donde se resalta el otorgamiento de derechos de explotación de propiedad intelectual por 14 años (a pesar de las críticas de varios sectores académicos y empresarial quienes alegaron que la comercialización particular a lo largo del territorio nacional y en el 
extranjero tardaría más del periodo otorgado de explotación de un invento y/o procedimiento) y la imposibilidad de que extranjeros obtuviesen patentes en Estados Unidos (Jaffe y Trajtenberg, 2002).

En 1793 se confiere la ley de patentes que remplazó el estatuto nacional de 1790 que definía la patente como una manufactura, máquina o arte útil, así como una composición de índole musical o científica (Hurt, 1966).

También aplicaba una mejora a una máquina, manufactura o elaboración de arte lo que significaba en la práctica un limbo jurídico aplicable para cualquier modificación sensible al cambio original de invención (esta es la base de las dificultades actuales en el otorgamiento de patentes frente al derecho de libre competencia) donde, aun cuando la utilidad del producto fuese dudosa, la amplitud de la normativa permitía mayor facilidad en el otorgamiento de la patente. En 1836 se aprobó una reforma a la ley de patentes de 1793 de las cuales resalta la modificación de la oficina de patentes adscrita al Departamento de Estado, el conocimiento público de aplicación para el otorgamiento de una patente con el fin de evitar la aplicación de una patente de productos ya creados con el fin de descongestionar las solicitudes hábiles, la extensión por siete años de la duración de una patentes como respuesta a las múltiples críticas por la corta duración del derecho de propiedad y el permiso de aplicación y obtención de patentes nacionales por parte de extranjeros (Gould y Gruben, 1995).

En ese sentido, entre 1790 y 1793 solo se otorgaron 57 patentes, mientras que a partir de 1793 hasta 1836 se otorgaron más de 10.000 patentes debido a la amplitud y ambigüedad de la norma, muchas de ellas de baja calidad y sin utilidad práctica (Watson, 2013, p. 124).

Esta situación provocó un aumento sustancial en el número de demandas entre los dueños de las patentes y las empresas que desconocían la existencia de patentes frente a productos que consideraban innovadores. El número de solicitudes de patentes sobrepasó la capacidad de trámite de la recién creada Oficina de Patentes y Marca Registrada la cual en su momento no contaba con capacidad administrativa, financiera y de personal para tramitar todas las solicitudes (WIPO, 2013). En 1849 la oficina de patentes pasó a la jurisdicción del Departamento del Interior. En 1952 la estructura básica de la ley de patentes fue modificada resaltando la 
necesidad de descripción del inventor no solo de su invento, sino también la descripción de la base de su aporte a la sociedad.

Lo anterior con el fin de no otorgar patentes a productos obvios donde el solicitante de la patente se aprovechase de un procedimiento o producto particular de un campo específico del conocimiento. En 1982 se cré la Corte de Apelaciones del Circuito Federal con el fin de atender únicamente los casos donde la violación de una patente, así como la reclamación por su no otorgamiento se resuelva de forma rápida y especializada.

En 2011 se promulgó la ley de inventos Leahy-Smith cambiando el sistema de patentes donde se prioriza al solicitante que patente primero un invento antes que su creador (Cohen et al., 2000); aun cuando este no haya patentado el invento o procedimiento. Finalmente, después de la adopción del documento declaratorio de la Ronda de Uruguay por medio del cual el GAAT pasó a ser la OMC, Estados Unidos amplió el periodo de beneficio de explotación de patente de 17 a 20 años a partir de su obtención.

En materia de fomento del libre mercado, en 1890, se promulga la ley Sherman anti-monopolio con el fin de restablecer el equilibrio de mercado a partir de la garantía de condiciones equitativas que fomenten la competencia que beneficia al consumidor final (Denison, 1979).

Lo anterior como respuesta a la alta concentración de industrias en manos de un competidor (caso Estándar Oil de John Rockefeller) a partir de la regulación de precios de insumos con el fin de evitar la creación de carteles, acuerdos extraoficiales, regulación del poder de mercado de un competidor en comparación con el resto y el no otorgamiento y/o defensa de patentes de inventos de gran alcance en cuanto a utilidad se refiere para la población estadounidense.

La ley antimonopolio fue el resultado de constantes críticas al derecho exclusivo de explotación de inventos o procedimientos en un entorno de crisis económica donde se tenía una percepción general de que las patentes limitaban la competencia y aumentaban los costos fijos de producción y distribución de mercancías y/o servicios. Sin embargo, las patentes no se vieron en amenaza de desaparecer, más aún, el número total de otorgamiento aumentó de forma exponencial. La gráfica 1 expone el crecimiento de solicitudes de patentes en Estados Unidos durante el periodo 1970-2012. 


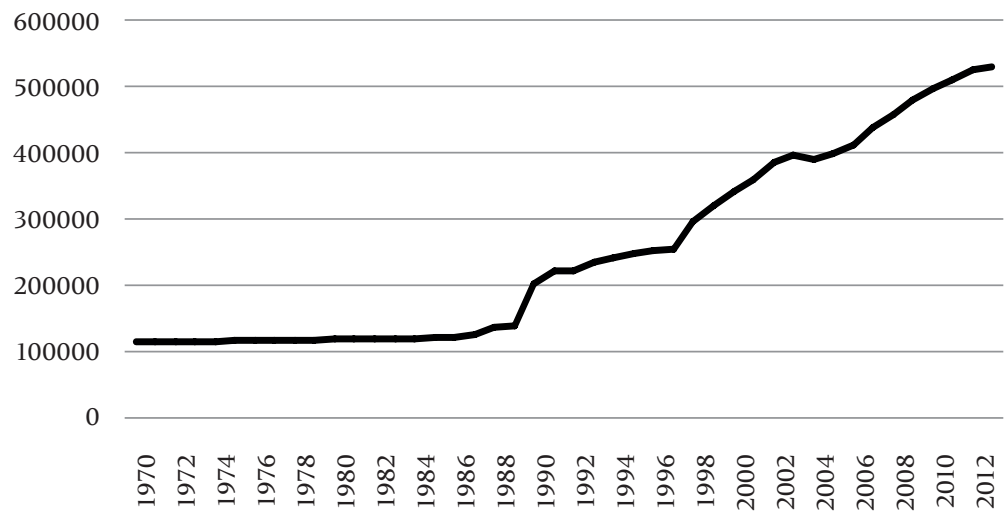

Gráfica 1. Cantidad de solicitudes de patentes por parte de nacionales y extranjeros en Estados Unidos, 1970-2012

Fuente: PWC (2014).

La posibilidad de que extranjeros soliciten patentes en suelo norteamericano ha aumentado el número de solicitudes de forma notable durante los últimos años en diversos campos del conocimiento siendo el sector de la tecnología computacional y médica líderes. La gráfica 2 muestra la distribución porcentual de las solicitudes de patentes por sectores productivos para el año 2013.

11\% Tecnología computacional

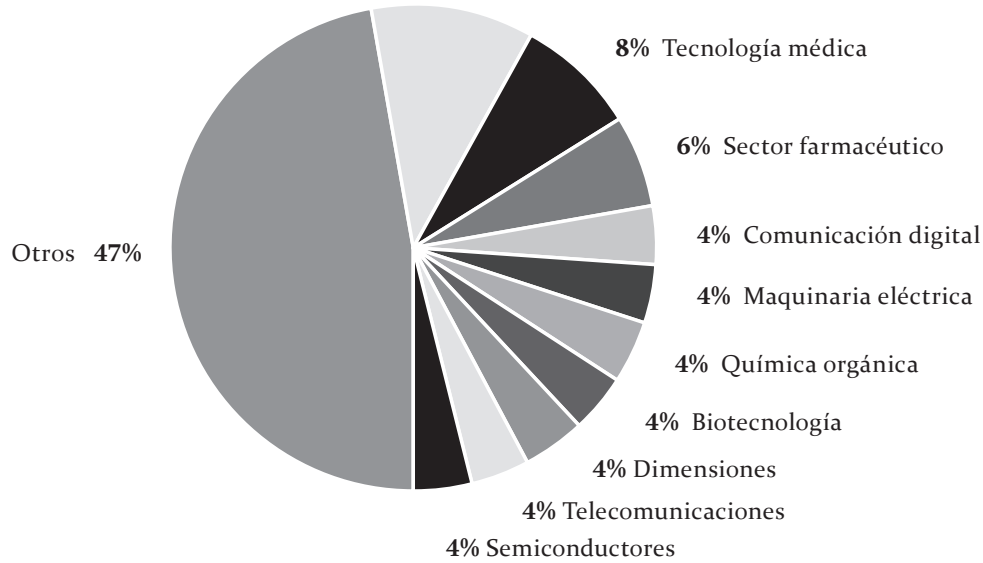

Gráfica 2. Distribución de solicitudes de patentes por sectores productivos en 2013

Fuente: PWC (2014). 
La forma en que la oficina de patentes otorga el beneficio a determinado solicitante es un tanto compleja y requiere una precisión absoluta en la presentación de la solicitud. Como primer requisito una patente se otorga cuando no es obvio el resultado obtenido (existe una ambigüedad en el concepto de obviedad que crea un vacío legal), es considerado novedoso y útil, a su vez tiene que ser descrito minuciosamente. Dentro de esta descripción se debe incluir el nombre del invento, resumen del mismo, procedimiento que se siguió para llegar a este, gráficos, dibujos y todo aquello que permita diferenciar el invento de algún otro y que muestre características únicas que se obtienen por medio del procedimiento descrito. Se manejan tres tipos de patentes que se complementan entre sí: las patentes de diseño, las patentes de utilidad y las patentes vegetales. Para efectos de la presente investigación, se explica la patente de diseño porque su facilidad en la obtención y la disminución en rigor para su examinación resulta fundamental para la emanación de este derecho que fomenta información asimétrica y, por consiguiente, una mayor propensión a litigios para su defensa.

Las patentes de diseño se utilizan básicamente para productos manufacturados donde se le otorga al inventor la garantía de su autoría, las patentes de utilidad permiten una mayor protección a nuevos inventos o procesos, son generalmente usadas cuando se mejora algo ya existente, así como el otorgamiento del derecho de explotación de su invento y/o procedimiento.

En Estados Unidos la patente de diseño es una de las modalidades previstas de protección cuya característica principal es la protección única soporte tangible del artículo en cuestión (PWC, 2014) y no la estructura y funcionamiento para el cual fue diseñado. Según Watson (2013) la patente de diseño protege de manera explícita la configuración de un artículo, su ornamentación superficial (no puede separase del artículo al que se aplica con lo cual no existe una patente de diseño por sí sola), así como la combinación de la configuración y la superficie de ornamentación. Al proteger únicamente la apariencia del artículo y no su funcionalidad, cuenta con mayor facilidad de obtención ya que no es necesario el pago por concepto de mantenimiento, solo es necesaria una reivindicación, el periodo de prioridad internacional es de seis meses y no 12 como en el caso de las patentes de utilidad, no se encuentran sujetas a publicación (Protectia, 2016). 
Expuesto lo anterior se procede a estudiar la escasez de análisis de obviedad y novedad de una solicitud de patente y las consecuencias en inversión, producción, distribución, comercialización y consumo de bienes tangibles que firmas y personas desean establecer en un entorno de incertidumbre y posterior información asimétrica.

Seguidamente se analizan las consecuencias en consumo e inversión de la obtención de patentes en un entorno de limitación de recursos monetarios y equipos especializados por parte de la autoridad encargada de estudiar las solicitudes de patentes sobre el beneficio del consumidor y las perspectivas de inversión en creación de nuevos bienes por parte de firmas de gran y mediano tamaño, así como fondos de inversión de capital privado. Finalmente se ofrecen algunas recomendaciones para mejorar la concesión de patentes con el fin de asegurar la libre actuación de agentes económicos en un entorno de mayor certidumbre empresarial.

\section{Escasez en análisis de obviedad y novedad}

Según Buchanan (1986) al considerarse la patente como una institución jurídica dada, existe la concepción que la equipara más como un derecho personal que como un monopolio de derecho dadas las implicaciones negativas para competencia en materia de incertidumbre en cuanto a la creación de nuevos bienes y limitación de flujo de información. En ese sentido, el derecho exclusivo del inventor de otorgar licencias limita en materia de acceso y costes indirectos a la sociedad del goce y disfrute de la proliferación de inventos que suponen un beneficio colectivo (Pérez y Soto, 2013). Si a esto se suma la imposibilidad de conocimiento de productos que violen una patente resulta en una distorsión del libre funcionamiento del mercado donde se desvía el ejercicio de la función empresarial impidiendo la solución de problemas cotidianos y especializados, además del acceso a sus soluciones.

Si bien la protección de derechos de propiedad intelectual es necesaria, el otorgamiento superfluo de patentes a inventos considerados obvios como resultado de una limitación en el estudio de patentabilidad producto del desborde en la capacidad 
de respuesta de la oficina de patentes, produce una incertidumbre continua a empresarios que buscan suministrar bienes y servicios innovadores al público que desconocen la existencia de patentes de productos inventados y sacados al público (Cooper, 1938, p. 805).

El resultado es el desincentivo a crear bienes considerados innovadores por miedo a acciones legales y/o pago de licencias que aumenta los costos totales de producción limitándoles su competitividad al interior del país. La causa principal del otorgamiento de patentes (de bajo valor agregado consideradas obvias) es la ausencia de un procedimiento normativoadministrativo con suficiente capacidad logística que permita la revisión del aporte de una patente luego de haberse concedido a su propietario (Benegas, 1999).

En la actualidad la Oficina de Patentes y Marca Registrada analiza y discute la patentabilidad de un producto únicamente con el aspirante mientras que terceros solo se involucran cuando tiene patentes en su poder y consideran que el aspirante puede incurrir en riesgo de violación de la patente existente previo envío de carta de protesta al director de la Oficina Federal (Foray, 1994).

Debido a la escasa participación de terceros en el proceso de otorgamiento de unas patentes, el Congreso aprobó la Ley de Procedimiento Limitado que permite a terceros participar en la reexaminación de unas patentes una vez se otorgue en primera instancia. Sin embargo, la falta de especificidad de la norma en cuanto a la documentación probatoria y los términos del procedimiento que debe presentar el tercero en el proceso de reexaminación no ha incentivado el uso masivo de la ley (McKinsey, 2012).

La intención de un nuevo procedimiento administrativo de reexaminación de una patente es, por un lado, equiparar a terceros involucrados con el cuerpo administrativo y el solicitante otorgándole más participación que permita eliminar patentes consideradas obvias y sin un verdadero valor agregado para la utilidad y/o innovación en la sociedad. Por otro lado, al limitar la participación de terceros en la reexaminación de patentes se busca evitar el hostigamiento de los mismos hacia los solicitantes por un invento y/o procedimiento que represente una fuente de ingresos considerable. Sin embargo, aun cuando la vigencia de una patente se mantiene luego del proceso de reexaminación, estos pueden acudir 
a instancias judiciales para defender sus argumentos causando congestión en el sistema judicial, en el sistema de administración de patentes y una prolongación en la decisión final que cuesta recursos monetarios para efectos de inversión y otorgamiento de licencias.

A pesar de esto, el número de solicitudes de patentes se encuentra en aumento, así como el otorgamiento de las mismas dentro y fuera del territorio estadounidense. La gráfica 3 muestra las solicitudes de patentes y su otorgamiento al interior del país durante el periodo 2013-1999, mientras la gráfica 4 muestra las patentes solicitadas y otorgadas por no residentes en el mismo periodo.

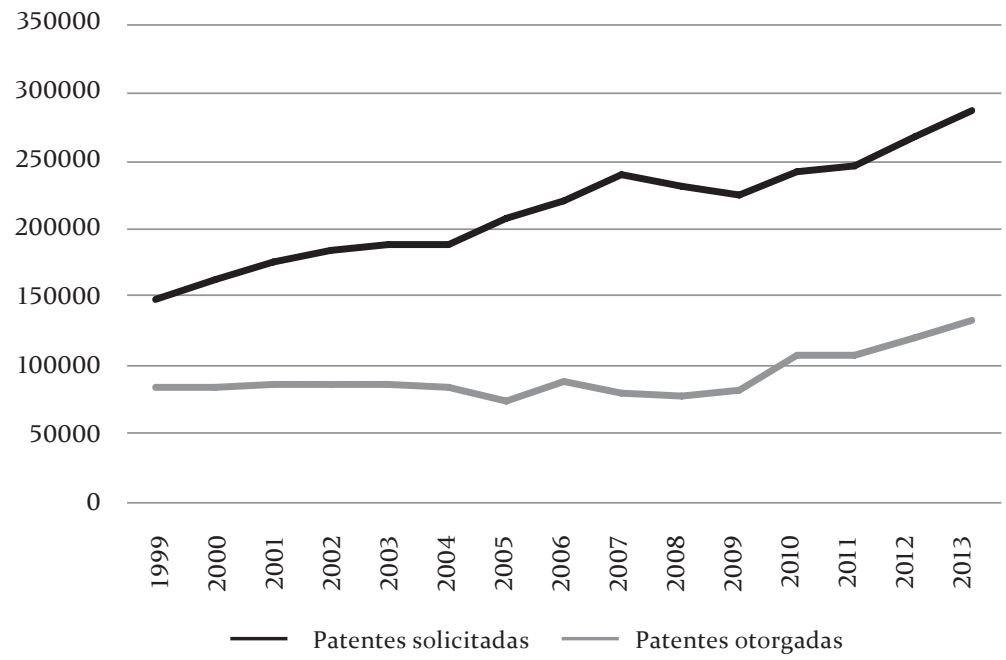

Gráfica 3. Patentes solicitadas vs. otorgadas en Estados Unidos por residentes, 1999-2013

Fuente: Cuban (2013).

Existe otra situación que permite el otorgamiento de patentes de baja calidad consideradas obvias que fomentan la incertidumbre a la innovación y desincentivan la producción de dichos bienes. Esta es la saturación de solicitudes de patentes y su reexaminación frente a los recursos monetarios, de personal y de equipos con el que cuenta la Oficina de Patentes.

En el año 2002 el Comité Asesor de Patentes (organismo adscrito a la rama legislativa con funciones de asesoría a la Oficina de Patentes) advirtió sobre la crisis que genera la escasez de fondos 
públicos para el funcionamiento general de la institución que resulta en una ampliación del periodo de respuesta frente a una solicitud de patentes, la reexaminación de una patente vigente, entre otros asuntos de su competencia.

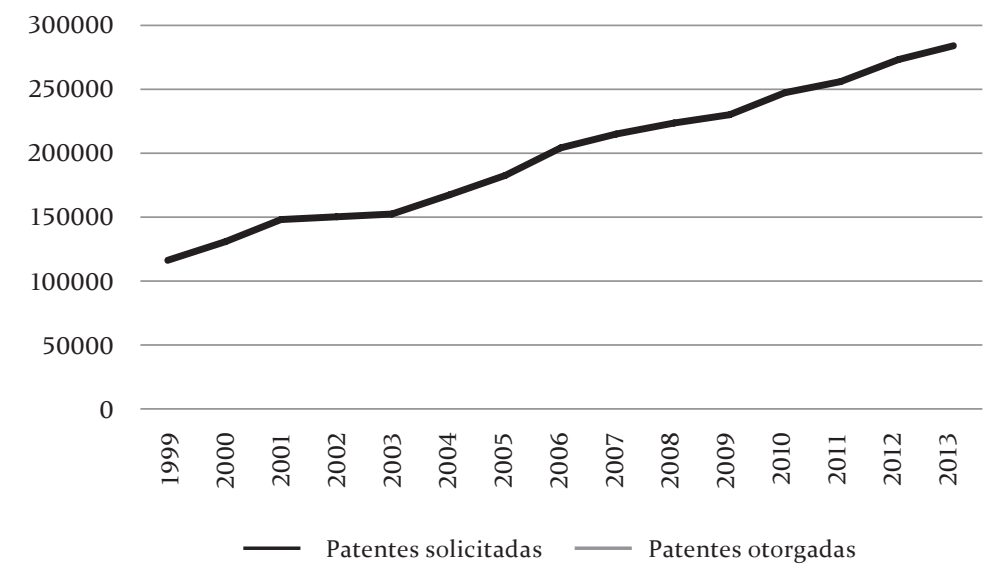

Gráfica 4. Patentes solicitadas vs. otorgadas en Estados Unidos por no residentes, 1999-2013

Fuente: WIPO (2014).

Esta situación provoca que las evaluaciones de novedad (entendida como el elemento de aporte no recurrente y repetitivo debidamente ilustrado) y el valor agregado de una solicitud de patente, así como la reexaminación sean de baja calidad permitiendo el otorgamiento de patentes a inventos y/o procedimientos considerados obvios por el sector privado y que generan incertidumbre en el funcionamiento general del mercado. La no obviedad de un invento se entiende como el desarrollo lo suficientemente técnico de un producto que amerita su patentabilidad (Mochón, 2012).

La aplicación adecuada de este principio de la ley de patentes es indispensable para prevenir el monopolio de derecho de explotación de un invento que popularmente puede ser considerado como obvio y sin valor agregado para la población, el cual puede encontrarse ya bajo dominio de la población (evitando litigios por violación de patente). Generalmente en caso de litigio por violación de patente cuando ésta se considera obvia, los jueces suelen solicitar una prueba comercial y otra de sugestión. 
El primero suele establecerse mediante pruebas de tiempo en donde un invento satisface una necesidad de largo plazo que otros no han podido satisfacer. Cuban (2013) menciona que una prueba comercial no implica la no obviedad de un invento que se dirija a un nicho específico de mercado que no satisfaga al grueso de la población, además que el mercadeo y campañas publicitarias pueden crear una necesidad en la población aun cuando el invento y/o procedimiento no sea objetivamente indispensable para el consumidor final.

La segunda prueba se basa en el análisis de no obviedad para un experto en el área en el cual se desarrolla el invento siendo la no obviedad como lo que no va más allá del progreso ordinario de la tecnología (CDIP, 2012), que se deduce de modo simple y lógico a partir del estado de la técnica de elaboración del invento y/o procedimiento.

Cohen et al. (2000) destacan que estas pruebas más la falta de capacidad operativa de la oficina de patentes frente al crecimiento interanual de las solicitudes dan como resultado el mantenimiento y vigencia de patentes de bajo valor agregado consideradas por el público en general como obvias, distorsionando la competencia interna. La gráfica 5 muestra el crecimiento de las patentes vigentes de residentes, no residentes y solicitadas en el extranjero durante el periodo 1999-2013.

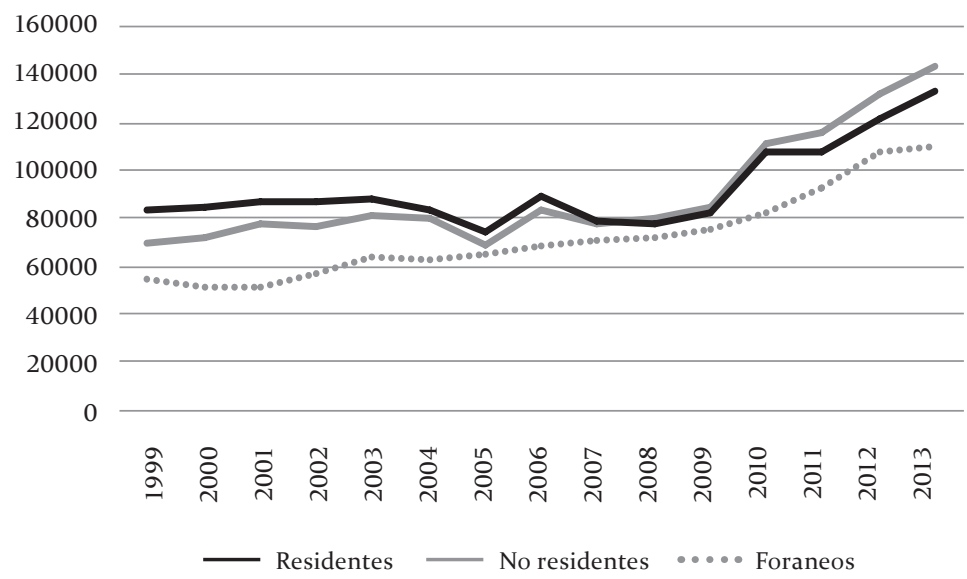

Gráfica 5. Crecimiento de las patentes vigentes de residentes, no residentes y solicitadas en el extranjero, 1999-2013

Fuente: WIPO (2014). 


\section{Información asimétrica e incertidumbre}

El establecimiento de baja calidad en diversas áreas del conocimiento representa en sí un perjuicio neto para el consumidor que busca mejorar sus niveles de utilidad a través de la adquisición de bienes y servicios.

Según Bessen (2014) los litigios por concepto de infracciones a patentes de diseño y utilidad han impactado en una disminución del $48 \%$ de inversión en investigación y desarrollo de industrias variadas con disponibilidad de capital de riesgo, 19\% en investigación y desarrollo, gastos operacionales para firmas pequeñas y $14 \%$ de inversión restante.

La patente (principalmente de productos y/o procedimientos considerados obvios) es en sí misma un derecho monopolístico que les permite a los propietarios un dominio de mercado a través del otorgamiento de licencias que termina en muchos casos en un incremento de precios del producto y/o servicio para el consumidor final (Pérez y Soto, 2013, p. 307).

Desde la perspectiva de libre mercado enmarcado como principio fundamental de la actividad económica libre, este derecho monopolístico es en sí violatorio del principio de libre competencia enmarcada en las ciencias económicas en esquemas de economías de mercado, aun cuando en la normativa estadounidense no exista el concepto de monopolio entorno a la figura de la patente. Los resultados son la inaccesibilidad de productos (en muchos casos de primera necesidad como medicamentos de mayor concentración química que tengan efectos más intensos sobre un paciente) que limitan la elección del consumidor creando una situación de escasez ficticia donde, si bien el recurso se encuentra disponible para él, no puede adquirirlo debido a incrementos ficticios de precios por efectos diferentes en materia económica para su producción, distribución y comercialización (Pérez y Soto, 2013, p. 20).

Si bien en una economía regida por el principio de propiedad privada es importante salvaguardar el derecho de propiedad intelectual, este se encuentra en muchos casos por encima del beneficio global de acceso a bienes y servicios sin manipulación extraoficial de precios de los consumidores que se ven en muchos casos perjudicados por el monopolio legal de dominio de una idea y/o invento. 
En ese sentido, los principales costos de una patente para la sociedad consumidora, productora e inventora en Estados Unidos ronda los US150\$ mil millones, un incremento de más del 100\% respecto al 2009 según McKinsey y Co. (2012) siendo el estorbo al progreso técnico uno de los principales obstáculos a la innovación, la producción y comercialización de bienes y/o procedimientos anti-económicos por parte de competidores que buscan inventar alrededor de una patente existente para evitar litigios, y la distorsión de incentivos de la actividad inventiva hacia productos más fácilmente patentables resultando en productos de muy bajo valor agregado en muchos casos considerados obvios.

Sin embargo, los efectos perjudiciales de la obtención de patentes de alto y bajo valor agregado no solo se limitan al consumidor final sino también distorsiona toda la dinámica de principios empresariales donde una empresa y/o persona natural que oferta un artículo patentado opta por ceñirse, en muchos casos, únicamente a litigios por violación de su propiedad intelectual generando mayores ingresos por litigios ganados que por bienes adquiridos por consumidores.

Esta situación se presenta principalmente en empresas micro y de pequeño tamaño donde la violación de una patente por parte de otra de mayor tamaño y músculo financiero limita el accionar competitivo de la primera, optando por la vía legal como recurso único para obtener cuota de mercado.

Lo anterior se debe principalmente al dinamismo del sistema legal estadounidense y la utilización del litigio como método de resolución de conflictos que, si bien se acogen a los principios constitucionales internos, ciertamente desincentiva la competencia en términos económicos ya que los micro y pequeños empresarios ven en la demanda un camino más corto para acaparar mayor cuota de mercado de un bien o proceso. La gráfica 6 muestra el crecimiento sostenido de litigios por violación de derechos de propiedad intelectual en Estados Unidos durante el periodo 1991-2013.

El crecimiento sostenido a partir de 2011 se debe a la entrada en vigor de la reforma a la ley de patentes que abre la posibilidad de otorgar la patente al inventor que primero registre el invento y/o proceso y no al primero en inventarlo lo que disparó el número de litigios en más de $24 \%$. Los productos de consumo y biotecnología son los de mayor demanda representando un $17 \%$ y $14 \%$ del total de litigios respectivos. 


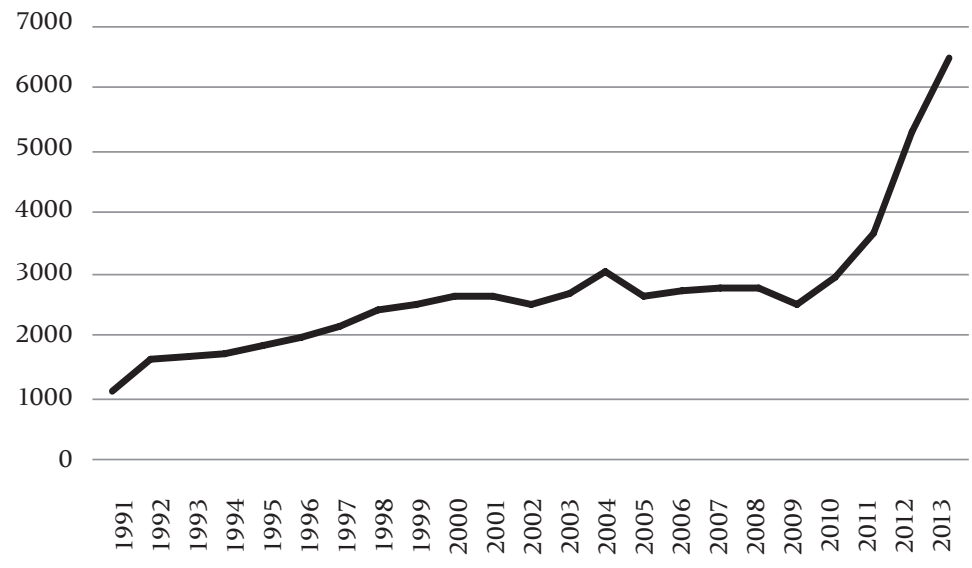

Gráfica 6. Crecimiento sostenido de litigios por violación de derechos de propiedad intelectual en Estados Unidos, 1991-2013

Fuente: PWC (2014).

La gráfica 7 expone la distribución porcentual de litigios para las diez industrias de mayor participación en la actividad económica estadounidense durante el periodo 1995-2013.

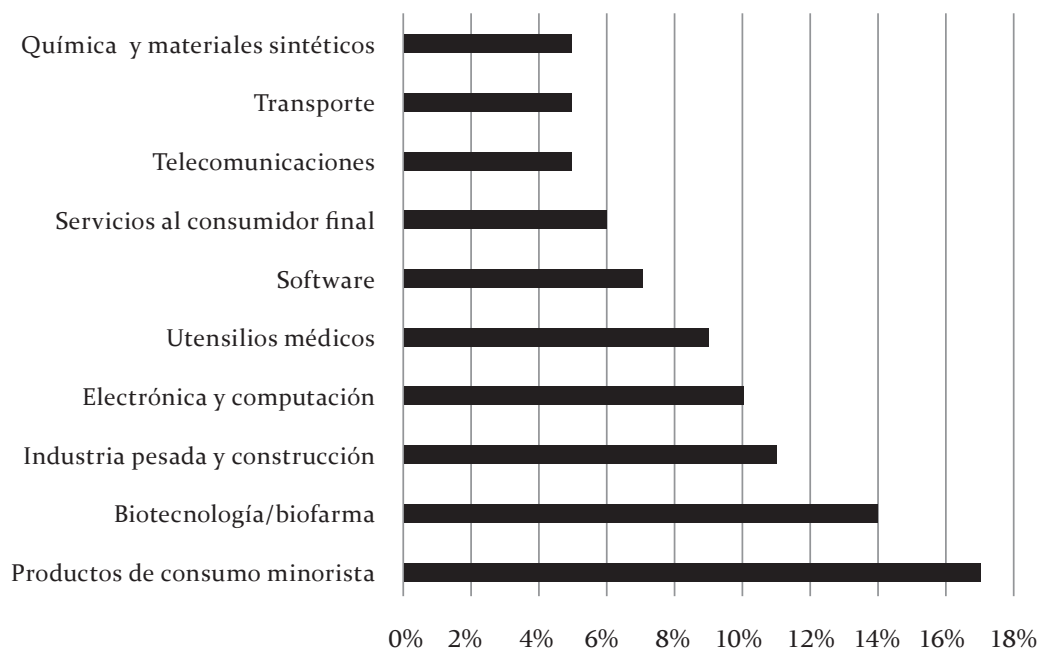

Gráfica 7. Distribución de litigios para las diez industrias de mayor participación en la actividad económica estadounidense, 1995-2013 Fuente: PWC (2014). 
En ese sentido el porcentaje de casos ganados por los dueños de patentes en litigios se encuentra alrededor del $35 \%$ para las diez principales industrias del país.

La gráfica 8 expone el porcentaje de casos ganados por dueños de patentes en litigios durante el periodo 1995-2013. La creciente utilización del sistema judicial estadounidense con respeto a la defensa del derecho de propiedad intelectual de productos y/o procedimientos complejos y considerados obvios ha prolongado el periodo promedio de resolución de litigios, así como ha incrementado el monto de indemnización monetaria afectado directamente por la prolongación de una decisión judicial.

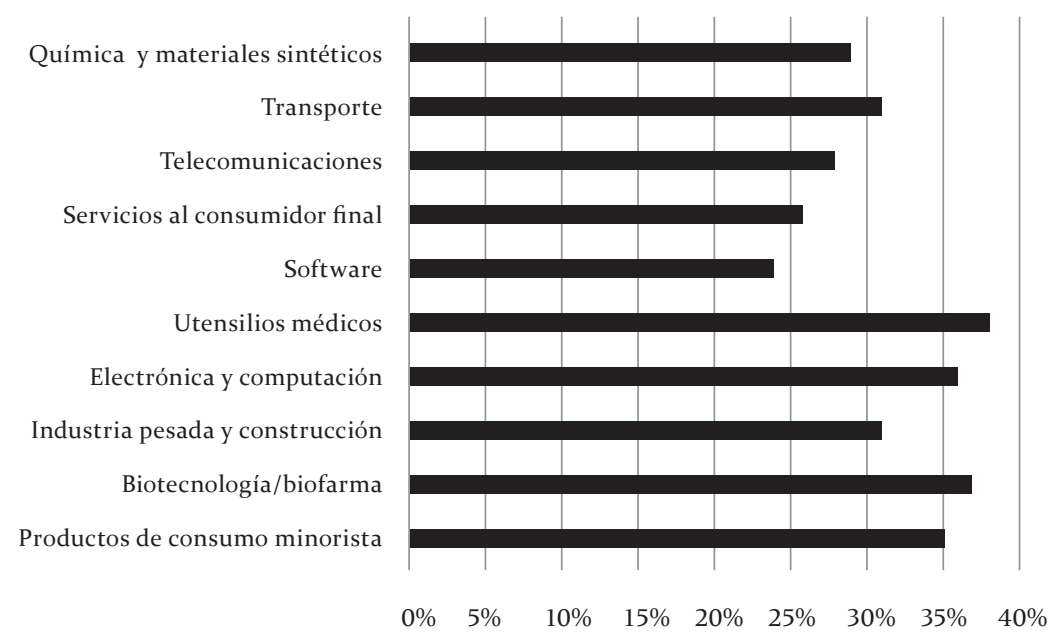

Gráfica 8. Porcentaje de casos ganados por dueños de patentes en litigios, 1995-2013

Fuente: PWC (2014).

La gráfica 9 expone en el eje izquierdo el monto promedio de indemnización en millones de dólares y en el eje derecho la duración promedio de litigio en años (dividido en cuatrienios desde 1995 hasta 2013). Nótese la correlación entre el crecimiento del periodo de tiempo de litigios en vigor hasta la fecha y el crecimiento en el monto de indemnización debido en muchos casos a la utilización indiscriminada del sistema legal para defender patentes en muchos casos consideradas obvias por la comunidad en general. 


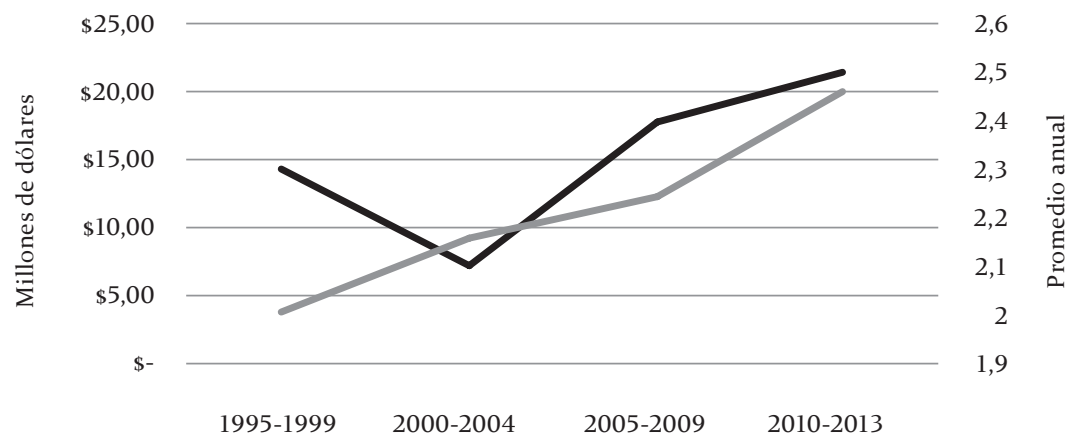

Monto promedio de indemnización en millones de dólares (izquierda)
— Duración promedio de litigio en años (derecha)

Gráfica 9. Monto promedio de indemnización en millones de dólares (izquierda), duración promedio de litigio en años (derecha), 1995-2013 Fuente: elaboración propia.

Por último, se exponen los montos promedios de indemnización en las diez industrias de mayor actividad económica estadounidense. Sobresale el sector biotecnológico y farmacéutico con multas alrededor de US\$20 millones en promedio debido a litigios de patentes sobre compuestos químicos en medicamentos comerciales y el sector de telecomunicaciones con alrededor de US\$22 millones por litigios alrededor de procesos y aplicaciones que involucran en más del $80 \%$ de los casos dispositivos móviles.

Sin embargo, también se destaca la industria de utensilios médicos con más de US $\$ 15$ millones en promedio en multas, electrónica y computación con más de US\$10 millones. Todo esto hace suponer que, si bien es indispensable la protección del derecho de propiedad intelectual materializado en litigios por patentes que distorsionan el principio de libre competencia y emprendimiento como resultado de la incertidumbre que genera el desconocimiento de la existencia de una patente sobre un invento o innovación en procedimientos de una empresa.

Para lograr mayor sinergia entre el mercado de patentes, sus autorizaciones y la no obstaculización de la libre competencia, además del mantenimiento del espíritu de innovación basado en la satisfacción del consumidor final es necesario establecer una serie 


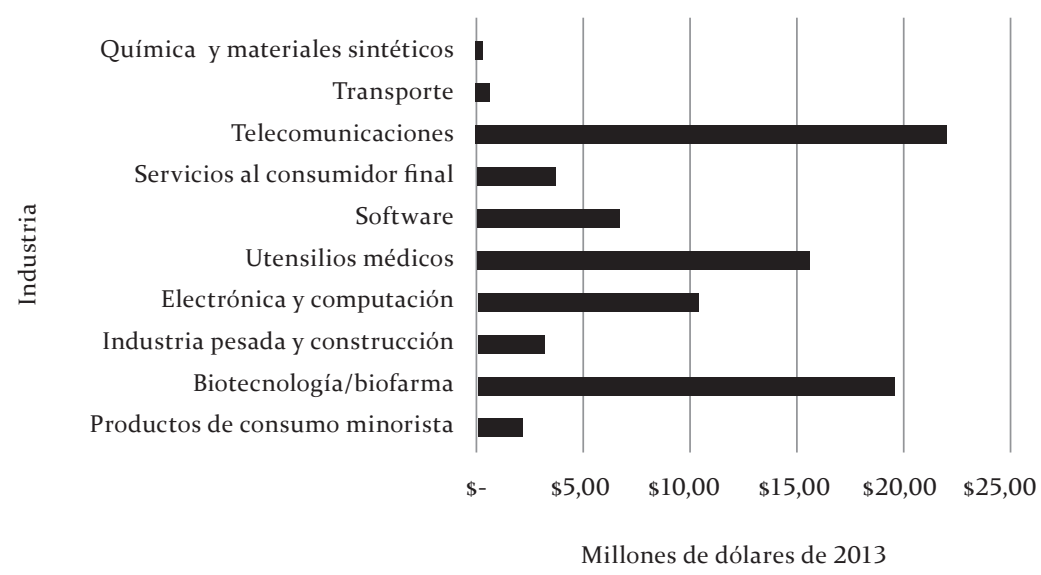

Gráfica 10. Promedio de multas por violación de derechos de propiedad intelectual por industria

Fuente: WIPO (2014). Global Innovation Index: Who is Leading Innovation? WIPO Publications.

de modificaciones tanto a los procedimientos de otorgamiento de patentes que pueden ser consideradas obvias, como en el proceso de litigios de las mismas en caso de presunción de violación del derecho de propiedad intelectual.

Es decir, si bien se busca que la patente como institución e instrumento de protección del derecho de propiedad intelectual se mantenga vigente en una economía de libre mercado, es necesario replantear el esquema de otorgamiento, reexaminación y litigio alrededor de esta.

Primero, es necesario reforzar el procedimiento administrativo de revisión, oposición y re-examinación de una patente donde se involucren terceros de manera que exista la posibilidad de evitar litigios en un futuro cercano por violación de derecho de propiedad intelectual. En ese sentido, es necesario especificar la prueba de sugestión con el fin de que se estudie si una patente se considera obvia más allá del conocimiento y opinión de un experto en la materia. Aun no existen estándares específicos sobre qué es el "progreso ordinario de tecnología" enmarcado en la ley de patentes que termina por distorsionar el mercado y afectar el principio de libre competencia mencionada en la ley antimonopolio. 
Segundo, es necesario dotar de recursos monetarios, bienes y equipo especializado a la Oficina de patentes para que pueda tramitar con mayor agilidad los procedimientos de solicitud, revisión y re-examinación de una patente. En la actualidad su presupuesto es limitado y no cuenta con suficiente personal especializado para reexaminar una patente, ni para tramitar la solicitud de su adquisición en periodos menores a un año. Finalmente, es necesario considerar las implicaciones negativas en cuanto a costos y acceso práctico de un invento y/o procedimiento al consumidor intermedio y final en el otorgamiento de una patente ya que los posteriores costos de uso de ésta por parte de la comunidad a la que va dirigida aumentaría estableciendo una discriminación de precios por medio de aumentos artificiales del costo de adquisición.

\section{Consideraciones finales}

El derecho de propiedad intelectual es característico de las economías de libre mercado donde se respeta la propiedad privada como fuente de libertades individuales y funcionamiento eficiente de los agentes económicos. Sin embargo, el otorgamiento indiscriminado de patentes por conceptos de creación y utilización sin una revisión rigurosa -que incluye una mayor participación de terceros en el proceso- promueve una distorsión de la competencia y un exceso de utilización del sistema legal que cuesta millones de dólares anuales a los contribuyentes en Estados Unidos. En ese sentido es indispensable reforzar los términos legales por los cuales se le otorgan patentes a solicitantes a través de especificaciones normativas procedimentales en las pruebas de comercialización y sugestión ya que en la actualidad estas cuentan con vacíos de los cuales no es posible diferenciar muchas veces un invento verdaderamente innovador, de otro que siga el principio de "progreso ordinario tecnológico".

Es indispensable reformar el otorgamiento de patentes a partir de la no obviedad de un producto basado en la participación limitada de terceros que permita alegaciones para evitar la concesión de productos que terminan perjudicando al consumidor final para no causar una distorsión de precios e incentivos a los 
productores y consumidores, donde los oferentes prefieren acudir al sistema legal para defender una idea y garantizar una participación en un nicho de mercado que competir de forma transparente.

\section{Referencias}

Allison, J. R., Lemley, M. A., \& Schwartz, D. L. (2014). Understanding the realities of modern patent litigation. Texas Law Review, 92, 21-56.

Almeida, P. (1996). Knowledge sourcing by foreign multinationals: patent citation analysis in the US semiconductor industry. Strategic Management Journal, 17, 155-165.

Benegas Lynch, A. (1999). Apuntes sobre el concepto de copyright. Libertas, 2, 121-147.

Bessen, J. (2014). The evidence is in: patents trolls do hurt innovation. Harvard Business Review, Noviembre, 1-4.

Bessen, J., \& Meurer, M. J. (2008). Patent failure: How Judges, Bureaucrats, and Lawyers Put Innovators at Risk. Princeton: Princeton University Press.

Bryant, T. L. (2011). America Invents Act: slaying trolls, limiting joinder. Tech., 25, 687.

Buchanan, J. (1986). Los Limites de la Libertad: Entre La Anarquía y El Leviatán. Barcelona: Liberty Fund.

Burk, D. L., \& Lemley, M. A. (2003). Policy levers in patent law. Virginia Law Review, 24, 1575-1696.

Burk, D. L., \& Lemley, M. A. (2009). The Patent Crisis and How the Courts can Solve it. Chicago: University of Chicago Press.

CDIP. (2011). Interface Between Exhaustion Intellectual Property Rights and competition Law. Disponible en: http://jiel.oxfordjournals.org/ content/1/4/655.full.pdf+html (consultado el 13 de diciembre de 2014).

CDIP. (2011). Report on an Analysis of Economic/Legal Literature on Intellectual Property Rights: A barrier to Entry? Disponible en: http://papers.ssrn. com/sol3/papers.cfm?abstract_id=723321 (consultado el 4 de Marzo de 2015).

Chien, C. V. (2008). Patently Protectionist? An empirical analysis of patent cases at the international trade commission. William $\mathcal{E}$ Mary Law Review, 50, 63.

Cohen, W. M., Nelson, R. R., \& Walsh, J. P. (2000). Protecting their intellectual assets: Appropriability conditions and why U.S. manufacturing firms patent (or Not). National Bureau of Economic Research, 4, 98. 
Cohen, W. M., Goto, A., Nagata, A., Nelson, R. R., \& Walsh, J. P. (2002). $\mathrm{R} \& \mathrm{D}$ spillovers, patents and the incentives to innovate in Japan and the United States. Research Policy, 31, 1349-1367.

Cooper, T. (1838). The Statutes at Large of South Carolina: Acts from 1716 to 1752. A.S. Johnstown: Johnston.

Constitutional Rights Foundation. (2011). The Origin Of Patents And Copyright Law. Washington D.C: Constitutional Rights Foundation.

Cuban, M. (2013). Lack of Innovation as a Consequence of Law Fragility (pp. 2327). Washington DC: Brooking Institution.

Curtis, G. T. (1849). A Treatise on the Law of Patents for Useful Inventions in the United States of America. Little Rock, U.S: Brown Stitzy.

Denison, E. F. (1979). Accounting for Slower Economic Growth. Washington D.C. U.S: Brookings Institution.

Director, A., \& Levi, E. H. (1956). Law and the future: Trade regulation. UL Rev, 51, 281.

Dupree, H. (1986). Science in the Federal Government: A History of Policies and Activities. Washington D.C, U.S: The Johns Hopkins University Press.

Eastman, S. E. (1949). Contributory infringement and the combination patent: a recognition of monopoly in unpatented elements created by combination patent enforcement. Michigan Law Review, 23, 183-200.

Foray, D. (1994). Production and distribution of knowledge in the new systems of innovation: the role of intellectual property rights. STI Review, 14, 98-99.

Gould. D \& Gruben, W. (1995). El papel de los derechos de la propiedad intelectual en el crecimiento económico. Economía Mexicana Nueva Época, 4, 23-25.

Hayek, F. A. (1948). 'Free' Enterprise and Competitive Order. [1947], en Individualism and Economic Order, Chicago: University of Chicago Press. 107-108.

Hurt, R. M. (1966). The economic rationale of copyright. American Economic Review, 56, 421-432.

Iwaisako, T., \& Futagami, K. (2003). Patent policy in an endogenous growth model. Journal of Economics, 78, 239-258.

Jaffe, A. \& Trajtenberg, M. (2002). Patents, Citations and Innovations: A Window on the Knowledge Economy. Massachusetts: MIT Press.

Janicke, P. M., \& Ren, L. (2006). Who wins patent infringement cases. AIPLA QJ, 34, 1.

Jasper, T. (2015). Software Patents: A One-Year Review of Alice v. CLS Bank. Journal of the Patent and Trademark Office Society, 97, 532-550.

Jeruss, S., Feldman, R., \& Walker, J. H. (2012). The America invents act 500: effects of patent monetization entities on U.S litigation. Social Policy Review, 21, 34-35. 
Khan, B. Z., \& Sokoloff, K. L. (2004). Institutions and democratic invention in 19th-century America: Evidence from "Great Inventors", 17901930. American Economic Review, 12, 395-401.

Khan, B. Z., \& Sokoloff, K. L. (1993). "Schemes of practical utility": entrepreneurship and innovation among "great inventors" in the United States, 1790-1865. The Journal of Economic History, 53(2), 289307, doi: https://doi.org/10.1017/S0022050700012924

Kitch, E. W. (1966). Graham v. John Deere Co.: New standards for patents. The Supreme Court Review, 43, 293-346.

Lanjouw, J. O., \& Schankerman, M. (2001). Characteristics of patent litigation: a window on competition. Journal of Economics, 31, 129-151.

Lemley, M. A. (2001). Rational ignorance at the patent office. Northwestern University Law Review, 95, 24-28.

Marks, D. H. (1985). Patent licensing and antitrust in the United States and the European Economic Community. Am. UL. Rev., 35, 963.

Mándi, A. (2003). Protection and challenge of pharmaceutical patents. Journal of Generic Medicines: The Business Journal for the Generic Medicines Sector, 1, 72-82.

McKinsey \& Co. (2012). Generating Value in Generics. McKinsey \& Co. Publications.

McGrath, R. J. (1990). Unauthorized use of patents by the United States government or its contractors, The. AIPLA QJ, 18, 349.

Mochon, A.l. (2012). Brief history of patents and trademarks in America. Brainstorming Pub. 21, 24-25.

Mokyr, J. (1990). The Lever of Riches: Technological Creativity and Economic Progress. Berlin, Germany: Oxford University Press.

Moore, K. A. (2001). Are district court judges equipped to resolve patent cases. Harv. JL Tech, 15, 1.

Moore, K. A. (2000). Judges, juries, and patent cases: an empirical peek inside the black box. Michigan Law Review, 3, 365-409.

Musungu, S. F. (2001). The right to health in the global economy: Reading human rights obligations into the patent regime of the WTO-TRIPS Agreement (Doctoral dissertation, Faculty of Law, Makerere University-Kampala, Uganda).

Neale, A. D. (1960). The antitrust laws of the United States of America: a study of competition enforced by law. Law Review, 42, 98 .

Noble, D. F. (1979). America by Design: Science, Technology, and the Rise of Corporate Capitalism. Oxford, U.K: Oxford University Press.

Patent Act of 1793, Ch. 11, 1 Stat. 318-323 (1793). Washington DC, U.S.: Patent and Trademark Office.

Patent Act of 1836, Ch. 357, 5 Stat. 117 (1836). Washington DC, U.S.: Patent and Trademark Office. 
Pérez y Soto, A. \& Flores, K. (2013). La patente como límite a la innovación tecnológica. Procesos de Mercado: Revista Europea de Economía Política, 10, 107-109.

Pérez y Soto, A. (2013). La Información Secuestrada: El Modelo de Servidumbre de Thomas Hobbes y Su Alternativa Liberal Austriaca en Friedrich August Von Hayek. (Tesis Doctoral, Facultad de Ciencias Jurídicas y Sociales, Universidad Rey Juan Carlos).

Penrose, E. (1974). La economía del sistema internacional de patentes. Barcelona: Siglo XXI.

Plant, [Sir] A. (1974). The Economic Theory Concerning Patents for Inventions. San Diego: Oruro Editions.

Porter, E. (1929). U.S. Patent No. RE17, 274. Washington, DC: Patent and Trademark Office.

Powell, T. R. (1917). The nature of a patent right. Columbia Law Review, 24, 663-686.

Protectia Patentes y Marcas. (2016). Qué es Una Patente de Diseño y Como Funciona. Disponible en: http://www.protectia.eu/ disenos-industriales/que-es-una-patente-de-diseno/ (consultado el 13/06/2016).

PWC. (2014). Patent litigation study as case volume leaps: damages continue general decline. Federal Register Monthly Review, 60, 12-13.

Rabinowitz, A. B. (2005). When does a patent right become an antitrust wrong? Antitrust liability for refusals to deal in patented goods. Rich. JL E Tech., 11, 7-8.

Rantanen, J., \& Petherbridge, L. (2011). Toward a system of invention registration: the leahy-smith America invents act. Michigan Law Review First Impressions, 110, 24-62.

Rich, G. S. (1942). Relation between patent practices and the anti-monopoly laws. J. Pat. Off. Soc'y, 24, 241.

Scherer, F. M. (2009). Political economy of patent policy reform in the United States. High Tech. L., 7, 167.

Sidak, J. (2013). The meaning of FRAND, part one: royalties. Journal of Competition Law and Economics, 9, 939-944.

Sokoloff, K. L. (1988). Inventive activity in early industrial America: evidence from patent records, 1790-1846. The Journal of Economic History, 48, 813-850.

Usselman, S. W. (1991). Patents purloined: railroads, inventors, and the diffusion of innovation in 19th-century America. Technology and Culture, 21, 1047-1075.

Walterscheid, E. C. (1995). Priority of invention: How the United States came to have a first-to-invent patent system. AIPLA QJ, 23, 263. 
Watson, J. (2013). A History of the United States Patent Office. Washington DC: Official Press C.O. 123-125.

WIPO. (2014). Global Innovation Index: Who is Leading Innovation? WIPO Publications. Disponible en: https://www.globalinnovationindex.org/ content.aspx?page=press-release (consultado el 29 de mayo de 2015).

Zahralddin, R. X. (1992). Effect of broad patent scope on the competitiveness of United States industry. Del. J. Corp. L., 17, 949. 